\title{
Vibration Damping Performances of Buildings with Moving Façades Under Harmonic Excitation
}

\author{
Giulia Di Giovanni ${ }^{1} \cdot$ Davide Bernardini $^{1}$ (I)
}

Received: 10 July 2020 / Revised: 3 September 2020 / Accepted: 4 September 2020

(c) The Author(s) 2020

\begin{abstract}
Background Façade technologies are in continuous evolution and the idea to realize buildings equipped with cladding systems capable to undergo significant displacements relatively to the main structure has been considered by many authors as an opportunity to improve their vibration performances.

Method From a structural dynamics viewpoint, a building with a monolithic Moving Façade is essentially the same thing as a building with a Tuned Mass Damper. However, in the presence of excitations directly acting on the external surface of the building, there may be significant diferences of behavior. In this work, a first step towards a systematic comparison between the performances of buildings with Moving Façades and Tuned Mass Dampers is carried out in the simplest setting of 2 degrees of freedom modeling and harmonic excitation.

Results Despite the deceptive simplicity of the setting, some of the aspects related to the potential applicability of moving façades to vibration damping and the correlated limitations are discussed and critically analyzed. The analyses show that, depending on the tuning of the system, monolithic Moving Façades could effectively act as vibration absorbers with a potentially high efficiency. However, it turns out that good performances could be realized at the price of extremely large displacements of the façade. The possibility to pursue potential applications of this type of systems seems therefore to be subordinated to the search of solutions to limit such displacements within functionally acceptable ranges.
\end{abstract}

Keywords Double-skin façades $\cdot$ Moving façades $\cdot$ Cladding systems $\cdot$ Vibration damping $\cdot$ Tuned mass dampers

\section{Introduction}

Modern buildings are conceived as systems capable to perform complex functions that require them to comply with articulated and stringent requirements.

With the emergence of energy-consumption reduction as a major national concern, the search for better approaches and strategies in ameliorating energy efficiency of buildings is rapidly increasing [1]. One of the most important methods of saving energy in a building is by carefully designing its façade [2]. For this reason, innovative building façade concepts are becoming more and more relevant. An advanced cladding system should, therefore, allow for a comfortable

Davide Bernardini

davide.bernardini@uniroma1.it

Giulia Di Giovanni

giulia.digiovanni@uniroma1.it

1 Department of Structural and Geotechnical Engineering, Sapienza University of Rome, Rome, Italy indoor climate, sound protection and good lighting, while minimizing the demand for auxiliary energy input $[3,4]$.

In this context, Double-Skin Façades (DSF) systems offer great potentialities and are becoming increasingly popular among architects and engineers. A DSF is a system consisting of two glass skins detailed in such a way to create an air layer around the building. When properly designed in terms of size and ventilation, the air cavity can act as a thermal buffer capable to reduce problems such as undesired heat gain during the heating season and heat loss during the cooling season [5]. For the above reasons, the level of interest in DSF has grown rapidly over the last years as one of the best options in managing the interaction between the outdoors and the internal spaces while providing architectural flexibility to the design, especially in office buildings.

A further aspect related to the engineering and technology of cladding systems is related to the more stringent requirements that tall buildings are deemed to satisfy in terms of resistance to exceptional actions like blasts or impacts. Also 
in this context, the design of façades plays a crucial role to improve the general building performances [6].

In the past, façades were passive systems rigidly fixed to the structure and designed to shape the architectural appearance of the building and act as a barrier against climatic actions. The only displacements allowed were those needed to accommodate thermal expansion and local deformations due to gravity and wind actions. In the current practice, façade technology might be considered mature enough to cope with adaptive envelopes capable to interact with the environment and adapt their behavior to the outdoor climate, user needs or environmental actions [7]. Therefore, the possibility of cladding systems undergoing displacements relative to the main structure through suitable sliding connections can be considered as technically feasible.

The idea to exploit cladding displacements to improve building performances with respect to blasts and extreme seismic excitations goes back at least to [13] and led several authors to evaluate the possibility to design moving connections with relevant energy-dissipation capabilities (see, e.g., $[14,15]$ among many others).

In the context of buildings with DSF, Moon [8] proposed to exploit relative displacement between the façade and the main structure to improve the dynamical behavior of tall buildings subject to wind actions. This idea has then been further explored in [9-12]. In this concept, the mass of the façade is used to reduce vibrations of the main structure in a way conceptually similar to tuned mass dampers (TMD) but without the need to endow the building with additional mass and with the advantage of saving useful space for the allocation of vibration damping devices.

From a structural dynamics point of view, a building endowed with a monolithic moving façade (MF) is similar to a building equipped with a TMD. When the structure is subjected to excitations like base motion, there is no conceptual difference. However, in the presence of wind actions or any other action that can be modeled by means of forces acting on the external surface of the building, the excitation is applied on the secondary mass instead of the main one. Therefore, the equations of motions of a building with MF or TMD are essentially the same, except for a different excitation. In spite of the substantial equality of the governing equations, the resulting behavior of the two systems may be rather different. The dynamical behavior of buildings with TMD is very well known since the 1960s and its main features are reported in virtually any textbook on structural dynamics. On the contrary, the behavior of buildings with MF did not receive similar attention, evidently due to the lack of potential applications. However, in view of the above-described recent progresses in façade engineering technology, it may be interesting to investigate the dynamical performances of buildings with MF subject to external forcing.

In the following, a first step in this direction is carried out by means of a systematic comparison between the vibration damping performances of buildings endowed with MF and TMD in the simplest setting of 2 d.o.f. models under harmonic excitation.

\section{Basic Modeling of MF and TMD}

Moving façades (MF) could be realized in various ways depending on the geometry of the building, by dividing in a suitable way the cladding surface. In the context of a first approximation modeling, the deformability of the moving blocks can be neglected and a building with a MF can be described by a multi d.o.f. oscillator, with a degree of freedom for each moving block. This can be seen in analogy with the difference between a basic Tuned Mass Damper (TMD) and multiple-TMD. Accordingly, the simplest model of a building equipped either with a monolithic MF or a standard TMD is a system composed by two masses $m_{1}$ and $m_{2}$ representing, respectively, the main structure and the secondary mass, connected by springs of stiffnesses $k_{1}, k_{2}$

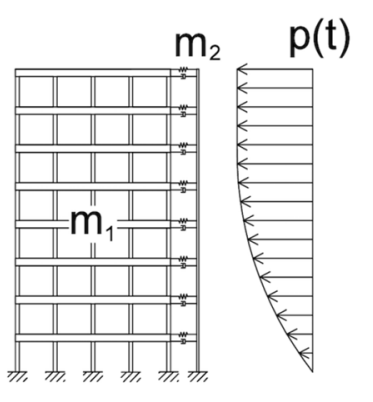

(a)
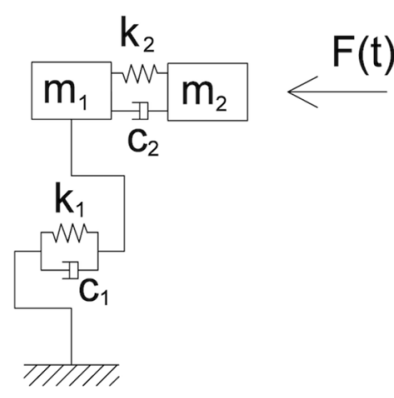
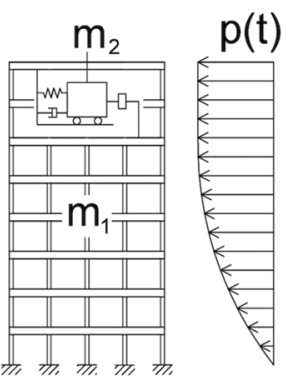

(b)

Fig. 12 d.o.f. modeling of a building equipped with a monolithic MF (a) and a single-mass TMD (b)

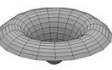


and viscous dashpots $c_{1}, c_{2}$, as shown in Fig. 1. Equations of motions can then be written in the form:

$\left\{\begin{array}{l}m_{1} \ddot{x}_{1}+\left(c_{1}+c_{2}\right) \dot{x}_{1}-c_{2} \dot{x}_{2}+\left(k_{1}+k_{2}\right) x_{1}-k_{2} x_{2}=f_{1} \\ m_{2} \ddot{x}_{2}-c_{2} \dot{x}_{1}+c_{2} \dot{x}_{2}-k_{2} x_{1}+k_{2} x_{2}=f_{2}\end{array}\right.$,

where $x_{1}$ and $x_{2}$ are the displacements of the two masses relative to the ground and $f_{1}, f_{2}$ forcing excitations on each degree of freedom. Clearly, when $f_{2}=0$, the system is a model for a standard TMD; whereas if $f_{1}=0$, it reduces to a model of a building with MF. Focusing attention on the response to harmonic excitation, it is useful to express it in complex form using a sans-serif font to distinguish complex from real numbers.

$\left\{\begin{array}{llrl}\mathrm{f}_{1} & =\mathrm{F}_{1} e^{i \Omega t} & \text { with } & \mathrm{F}_{1}:=F_{1} e^{i \Psi_{1}} \\ \mathrm{f}_{2}=\mathrm{F}_{2} e^{i \Omega t} & & \mathrm{~F}_{2}:=F_{2} e^{i \Psi_{2}},\end{array}\right.$

where $\Psi_{1,2}$ denote phase angles which describe the phase shifts between excitation and response.

Even if the solution of this system is very well known, its main aspects are recalled below to fix notation. Upon introduction of the standard notations

$\omega_{1}^{2}:=\frac{k_{1}}{m_{1}} \quad \omega_{2}^{2}:=\frac{k_{2}}{m_{2}} \quad \xi_{1}:=\frac{c_{1}}{2 m_{1} \omega_{1}} \quad \xi_{2}:=\frac{c_{2}}{2 m_{2} \omega_{2}}$,

the mass, natural frequency and forcing frequency ratios are defined as follows

$\mu:=\frac{m_{2}}{m_{1}} \quad f:=\frac{\omega_{2}}{\omega_{1}} \quad \rho:=\frac{\Omega}{\omega_{1}}$.

Natural frequency and mass ratios together determine the stiffness ratio via

$\sigma:=\frac{k_{2}}{k_{1}}=\mu f^{2}$

To evaluate the performances of the systems, it is useful to express the motion of secondary mass relatively to the main structure by introducing the variables

$u_{2}:=x_{2}-x_{1} \quad u_{1}:=x_{1}$.

So that system (1) can be rewritten in the form

$\left\{\begin{array}{l}\ddot{u}_{1}+2 \omega_{1}\left(\xi_{1} \dot{u}_{1}-\mu f \xi_{2} \dot{u}_{2}\right)+\left(\omega_{1}^{2}\right)\left(u_{1}-\mu f^{2} u_{2}\right)=\omega_{1}^{2} \frac{f_{1}}{k_{1}} \\ \ddot{u}_{2}+\ddot{u}_{1}+\left(2 \omega_{1} f \xi_{2}\right) \dot{u}_{2}+\left(\omega_{1}^{2} f^{2}\right) u_{2}=\omega_{1}^{2} \frac{f_{2}}{\mu k_{1}}\end{array}\right.$.

Assuming harmonic response

$\left\{\begin{array}{l}\mathrm{u}_{1}=\mathrm{U}_{1} e^{i \Omega t} \\ \mathrm{u}_{2}=\mathrm{U}_{2} e^{i \Omega t}\end{array}\right.$, substitution of (2) and (6) in (5) yields the following equations in the complex amplitudes of the response

$\left\{\begin{array}{l}\mathrm{Z}_{1,1} \mathrm{U}_{1}+\mathrm{Z}_{1,2} \mathrm{U}_{2}=\frac{1}{k_{1}} \mathrm{~F}_{1} \\ \mathrm{Z}_{2,1} \mathrm{U}_{1}+\mathrm{Z}_{2,2} \mathrm{U}_{2}=\frac{1}{\mu k_{1}} \mathrm{~F}_{2}\end{array}\right.$

where

$\mathrm{Z}_{1,1}:=\left[1-\rho^{2}\right]+i\left[2 \rho \xi_{1}\right]$

$\mathrm{Z}_{1,2}:=\left[-\mu f^{2}\right]+i\left[-2 \mu f \rho \xi_{2}\right]$

$Z_{2,1}:=\left[-\rho^{2}\right]$

$\mathbf{Z}_{2,2}:=\left[f^{2}-\rho^{2}\right]+i\left[2 \rho f \xi_{2}\right]$

Solving the system (7), complex amplitudes can be written in the following form which emphasizes the contributions of the parts relevant to TMD and MF in terms of the corresponding transfer functions

$\left\{\begin{array}{l}\mathrm{U}_{1}=\mathrm{H}_{1}^{\mathrm{TMD}} \mathrm{F}_{1}+\mathrm{H}_{1}^{\mathrm{MF}} \mathrm{F}_{2} \\ \mathrm{U}_{2}=\mathrm{H}_{2}^{\mathrm{TMD}} \mathrm{F}_{1}+\mathrm{H}_{2}^{\mathrm{MF}} \mathrm{F}_{2}\end{array}\right.$.

Each one of the four functions $\mathrm{H}$ can be seen as a special case of the formula

$\mathrm{H}:=\frac{\left[-B_{2} \rho^{2}+B_{0}\right]+i\left[B_{1} \rho\right]}{C_{0}+i C_{1}} \alpha$,

where the coefficients of the denominator are common to all cases

$C_{0}:=A_{4} \rho^{4}-A_{2} \rho^{2}+A_{0}$

$C_{1}:=-A_{3} \rho^{3}+A_{1} \rho$

with

$A_{0}:=f^{2}$

$A_{1}:=2\left(f \xi_{1}+\xi_{2}\right) f$

$A_{2}:=1+(1+\mu) f^{2}+4 f \xi_{1} \xi_{2}$,

$A_{3}:=2\left(\xi_{1}+(1+\mu) f \xi_{2}\right)$

$A_{4}:=1$

whereas, the coefficients of the numerator take different expressions in the four cases

$\begin{array}{lllll} & H_{1}^{\mathrm{TMD}} & H_{2}^{\mathrm{TMD}} & H_{1}^{\mathrm{MF}} & H_{2}^{\mathrm{MF}} \\ B_{0} & f^{2} & 0 & \mu f^{2} & 1 \\ B_{1} & 2 f \xi_{2} & 0 & 2 \mu f \xi_{2} & 2 \xi_{1} \\ B_{2} & 1 & 1 & 0 & 1 \\ \alpha & \frac{1}{k_{1}} & \frac{1}{k_{1}} & \frac{1}{\mu k_{1}} & \frac{1}{\mu k_{1}}\end{array}$

Substituting (8) into (6), the response to harmonic excitation can be written in the form 
$\mathrm{u}_{1}=H_{1}^{\mathrm{TMD}} \frac{F_{1}}{k_{1}} e^{i\left(\Omega t+\psi_{1}+\delta_{1}^{\mathrm{TMD}}\right)}+H_{1}^{\mathrm{MF}} \frac{F_{2}}{k_{1}} e^{i\left(\Omega t+\psi_{2}+\delta_{1}^{\mathrm{MF}}\right)}$

$\mathrm{u}_{2}=H_{2}^{\mathrm{TMD}} \frac{F_{1}}{k_{1}} e^{i\left(\Omega t+\psi_{1}+\delta_{2}^{\mathrm{TMD}}\right)}+H_{2}^{\mathrm{MF}} \frac{F_{2}}{k_{1}} e^{i\left(\Omega t+\psi_{2}+\delta_{2}^{\mathrm{MF}}\right)}$,

where $\delta_{1,2}$ denote phase angles.

In terms of the respective amplification factors, one has

$$
H_{1}^{\mathrm{TMD}}:=\sqrt{\frac{\rho^{4}+2 f^{2}\left(2 \xi_{2}^{2}-1\right) \rho^{2}+f^{4}}{C_{0}^{2}+C_{1}^{2}}}, \quad H_{2}^{\mathrm{TMD}}:=\sqrt{\frac{\rho^{4}}{C_{0}^{2}+C_{1}^{2}}},
$$

and

$$
\begin{gathered}
H_{1}^{\mathrm{MF}}:=\sqrt{\frac{\left(f^{2} \mu\right)^{2}+\left(2 f \mu \rho \xi_{2}\right)^{2}}{\mu^{2}\left(C_{0}^{2}+C_{1}^{2}\right)}}, \\
H_{2}^{\mathrm{MF}}:=\sqrt{\frac{\rho^{4}+2\left(2 \xi_{1}^{2}-1\right) \rho^{2}+1}{\mu^{2}\left(C_{0}^{2}+C_{1}^{2}\right)}} .
\end{gathered}
$$

\section{Vibration Damping Performance Indicators}

In general, the vibration amplitude of the main structure endowed with the secondary mass may be either bigger or smaller than the corresponding response of the bare main structure, which is given by

$H(\rho):=\frac{1}{\sqrt{\left(1-\rho^{2}\right)^{2}+\left(2 \xi_{1} \rho\right)^{2}}}$.

It is then useful to define a numerical indicator of the efficiency of the vibration damping of the added mass by means of the quantities

$$
\begin{aligned}
\eta^{\mathrm{TMD}}(\rho) & :=\frac{H(\rho)-H_{1}^{\mathrm{TMD}}(\rho)}{H(\rho)}, \\
\eta^{\mathrm{MF}}(\rho) & :=\frac{H(\rho)-H_{1}^{\mathrm{MF}}(\rho)}{H(\rho)} .
\end{aligned}
$$

Of course, if $\eta(\rho)>0$ the secondary mass acts as vibration damper for the main structure, whereas negative values of $\eta(\rho)$ indicate that the added mass acts as a vibration amplifier for the structure. The efficiency is not a constant, as the same secondary mass may act either as a vibration damper or amplifier depending on the excitation frequency. The frequency region where the secondary mass actually reduces vibrations of the main structure will be called vibration damping range (VDR). Similar indicators shall be defined in terms of other relevant response quantities like, for example, floor acceleration and interstory drifts; however, attention in the following will be restricted to response in terms of displacements.

\section{Tuning Ranges}

The response of the system exhibits qualitatively different types of behavior depending on the frequency ratio $f$ defined in (3) which determines the tuning between the natural frequencies of the independent vibrations of the two masses. To discuss such differences, it is useful to broadly distinguish three tuning ranges:

- tuned systems for values of $f$ close to 1 the peaks in the transfer functions are close to each other. In this situation, the stiffness ratio $\sigma$ is numerically close to the mass ratio $\mu$

- undertuned systems for lower values $f<1$ the resonance of the secondary mass takes place at lower frequencies than the main one. For fixed mass ratio, undertuning is obtained by means of softer connections;

- overtuned systems for higher values $f>1$, the resonance of the secondary mass takes place at higher frequencies than the main one. For fixed mass ratio, overtuning is obtained by means of stiffer connections.

Clearly, the same tuning level can be realized with different combinations of the mass and stiffness ratios which are related according to (4). For a given tuning, the response of the system is influenced by the damping ratios of both the main structure and the connections.

\section{Tuned Systems}

It is well known that for structures equipped with TMD and values of $f$ close to 1 , it is possible to activate a transfer of kinetic energy from the main structure to the secondary mass. Owing to this energy exchange, the TMD acts as an high efficiency vibration damper in a frequency interval centered about $\rho=1$.

Figure $2 \mathrm{a}, \mathrm{b}$ shows the transfer function $H_{1}$ for $f=1$ and various values of the mass ratio (with $\xi_{1}=0.03$ and $\left.\xi_{2}=0.10\right)$ superposed to the corresponding response of main structure without additional masses, plotted in red. Positive efficiency arises when the curve $H_{1}$ is below the red one. In the figure, a yellow colour highlights the region where the secondary mass acts as a vibration damper. Efficiency of TMD depends on the mass ratio and on the damping ratios $\xi_{1}$ and $\xi_{2}$ and there are various ways to optimize its performances [16]. For the chosen values of the parameters, the VDR extends approximately between 0.9 and 1.1 with a peak in efficiency of about 0.9 which increases with the mass ratio (Fig. 2c). 
Fig. 2 In the first row transfer functions $H_{1}$ for both TMD (a) and MF (b) with $f=1.00, \xi_{1}=0.03, \xi_{2}=0.10$ and $\mu \in[0.05,0.10]$. The isolated red curves show the amplification of the main structure without any added mass. In the second row: vibration damping efficiency $\eta$ for both TMD (c) and MF (d) where, for the sake of clarity, only values with positive efficiency are plotted. Blue curves corresponds to lower values of $\mu$, dark red curves to higher values of $\mu$
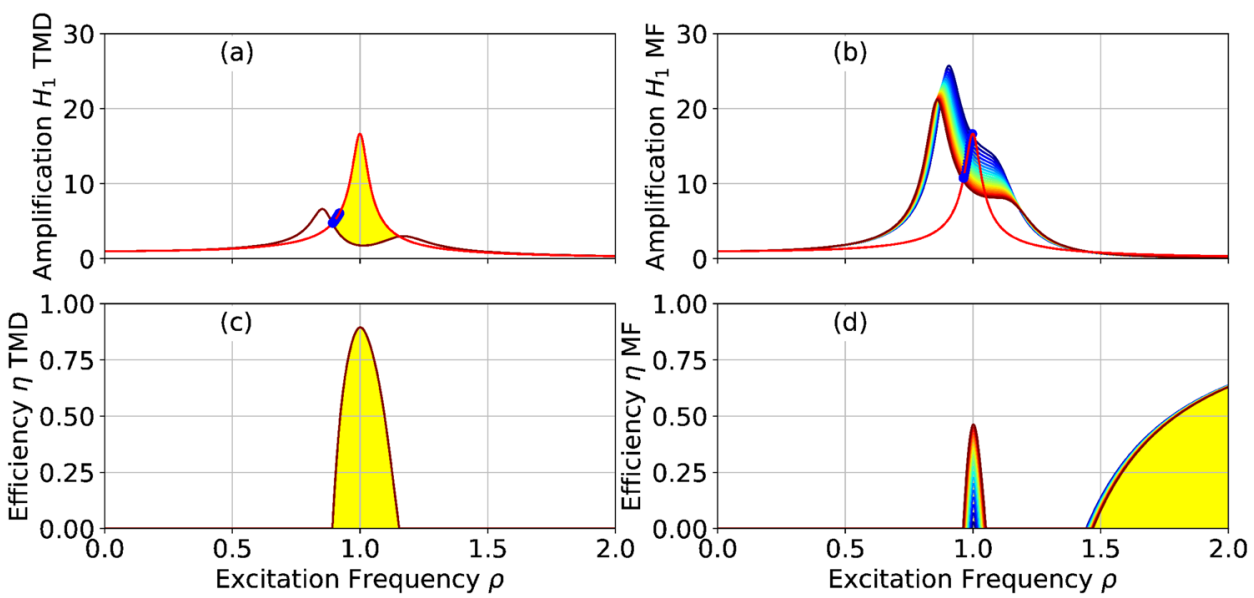

Fig. 3 Transfer functions $\mathrm{H}_{2}$ for the displacement of the secondary masses corresponding to Fig. 2. Blue curves correspond to lower values of $\mu$, dark red curves to higher values of $\mu$
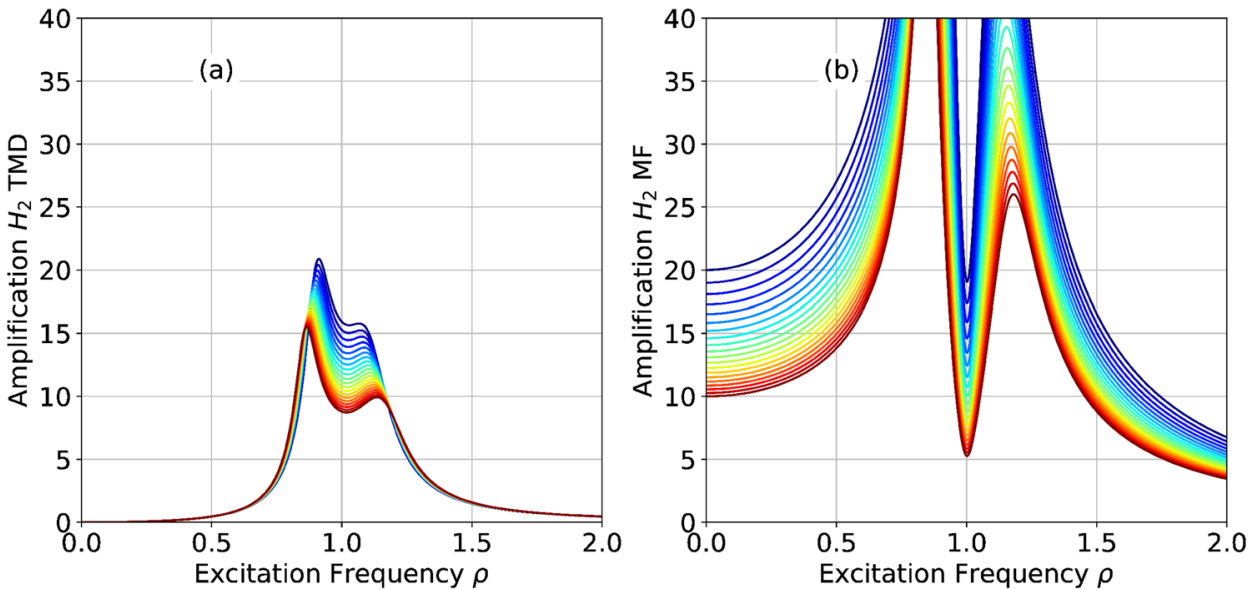

Under the same conditions, a tuned MF shows a lower efficiency near $\rho=1$ but tends to reduce systematically higher-frequency oscillations (Fig. 2d).

Figure 3 displays the amplification of the secondary mass in both the TMD (a) and MF(b) system under the same conditions as in Fig. 2. This shows that the displacement of the façade tends to be significantly bigger than the one of the mass in the TMD system, except in a narrow interval around $\rho=1$ and this effects strongly increases for lower mass ratios (blue curves).

\section{Undertuned Systems}

Decreasing the tuning ratio $f$ at fixed mass ratio $\mu$ corresponds to realize more flexible connections between the two masses.

In such conditions, the response of TMD and MF systems becomes significantly different.

As shown in Fig. 4, when the elastic coupling between the masses decreases (blue curves), the TMD progressively looses almost all of its vibration damping efficiency (Fig. 4c); whereas, on the contrary, MF gains a very high efficiency over a broad excitation frequency range (Fig. 4d). In Fig. 4a, b blue dots indicate the points where the amplification function $H_{1}$ intersects the red curve representing the main structure with no masses, hence defining the boundary of the VDR. The phenomenon is easily understandable by thinking that, for weaker and weaker couplings, the kinetic energy tends to remain on the mass where excitation is applied. Therefore, oscillations tend to localize in the main mass for the TMD and in the façade for MF systems.

Figure 5 describes in more detail the case of $f=0.4$ for various mass ratios and shows that, for this case of buildings with softer connections, a MF system is capable to realize excellent vibration damping performances for excitation frequencies close to $\rho=1$ (Fig. $5 \mathrm{~d}$ ), even better than the corresponding performances provided by the TMD in the case of $f=1$. This effect turns out to be essentially independent of the mass ratio and is still more pronounced by further decreases of $f$. 
On the other hand, a TMD system with softer connections between the masses has the property that the main structure oscillates essentially in the same way as if it was not connected to the secondary mass.
The localization of the oscillations in the secondary mass of a MF system with softer connections has a strong effect in the vibration damping efficiency but takes places at the price of higher displacements of the secondary mass.
Fig. 4 Transfer functions $H_{1}$ (a, b) and vibration damping efficiency $(\mathbf{c}, \mathbf{d}) \eta$ for tuning ratio $f \in[0.2,1.0]$ and fixed mass ratio $\left(\mu=0.05, \xi_{1}=0.03, \xi_{2}=0.10\right)$ with blue curves corresponding to lower values of $f$
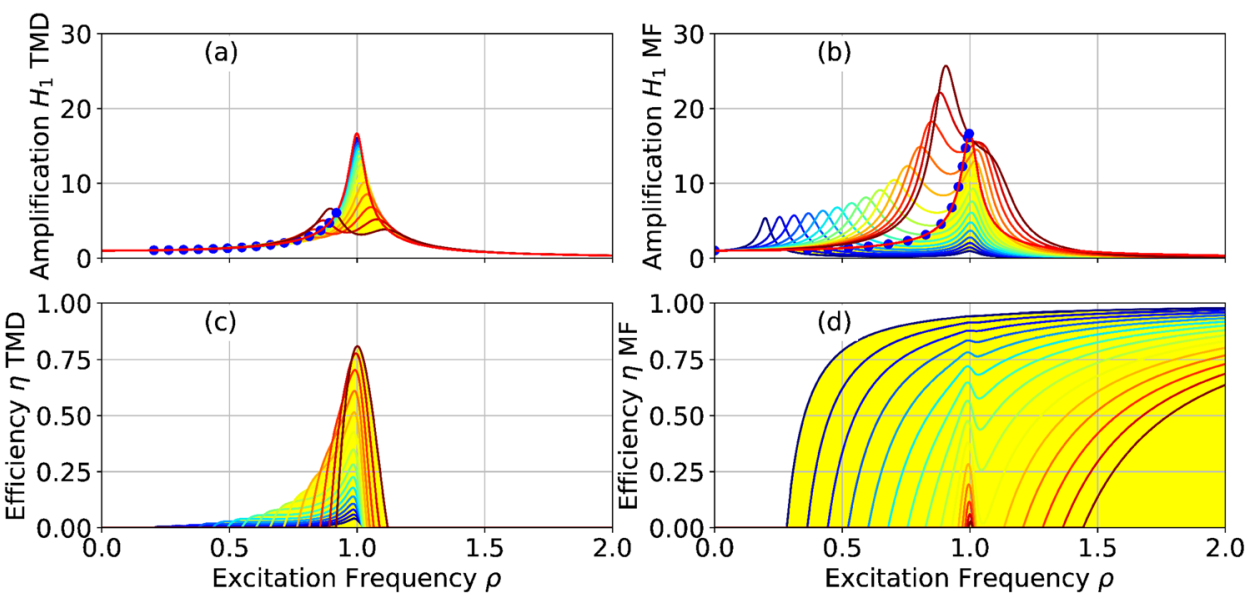

Fig. 5 Transfer functions $H_{1}$ (a, b) and vibration efficiency (c, d) for $f=0.4$ and various mass ratios $\mu \in[0.05,0.10]$ (other parameters as in Fig. 4) with a zoom of the interval $\rho \in[0.8,1.2]$ in the case of MF (b)
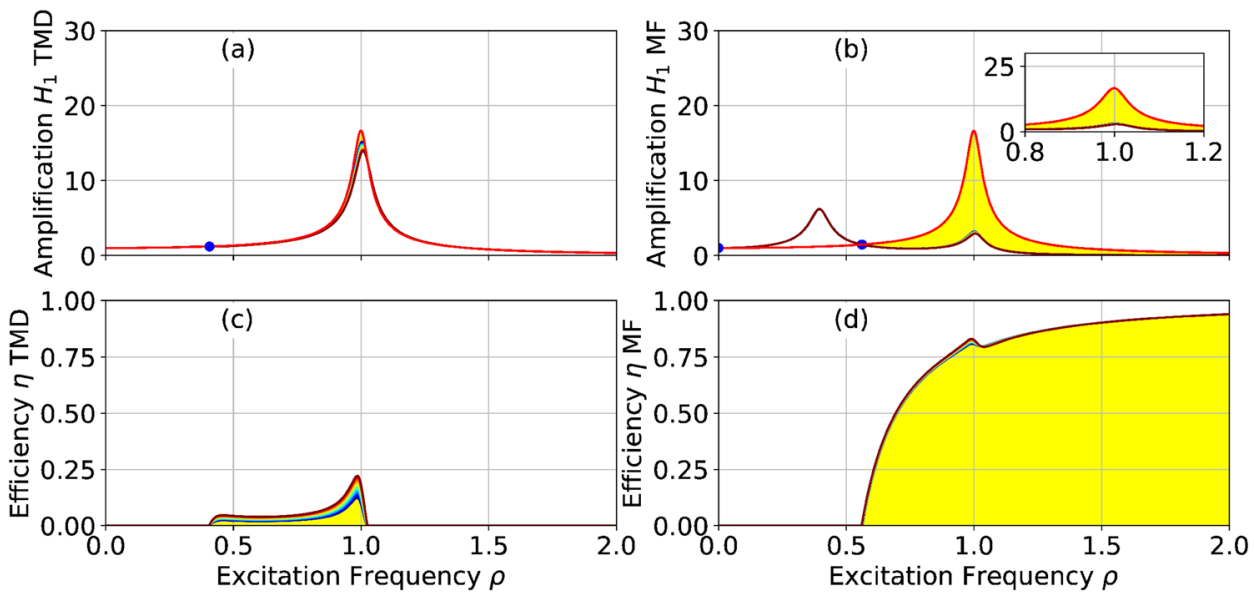

Fig. 6 Transfer functions $\mathrm{H}_{2}$ for $f=0.4$ (other parameters as in Fig. 5) with a zoom of the interval $\rho \in[0.8,1.2]$ in the case of MF (b). Blue curves correspond to lower mass ratios
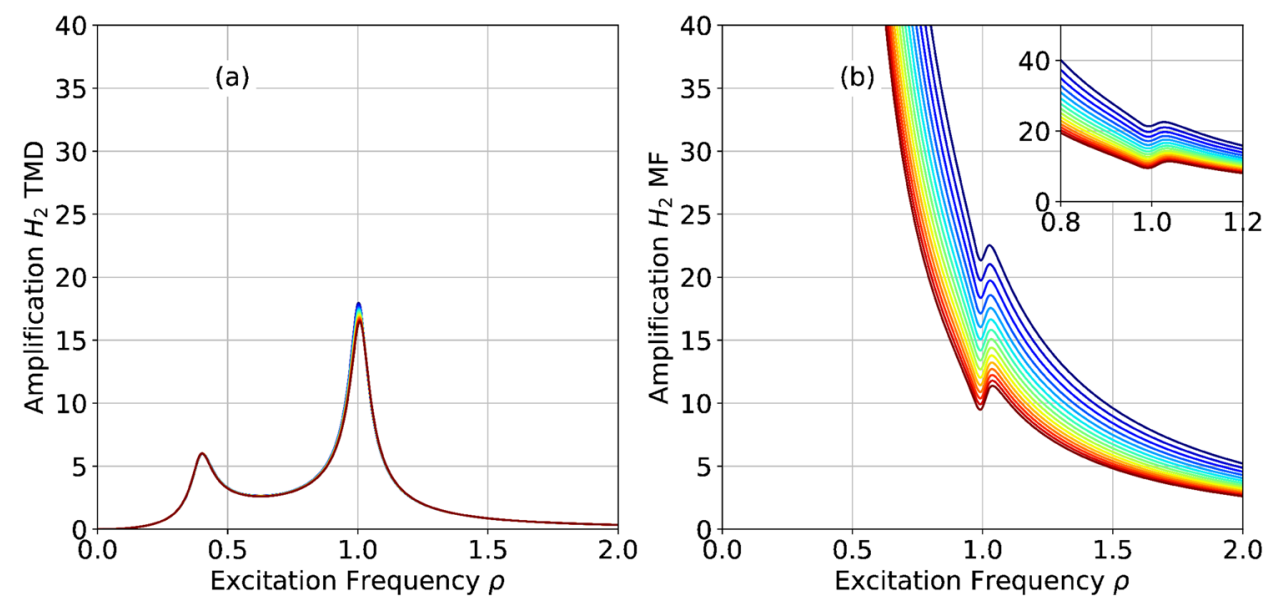
Figure 6 shows the transfer functions $H_{2}$ for $f=0.4$ in both TMD (a) and MF (b) systems for various values of the mass ratio. It is evident that displacements of the secondary mass becomes extremely large at lower excitation frequencies. However, if attention is focused on the region $\rho \in[0.8,0.10]$ (Fig. 6b) where vibration efficiency is still good, the values of the amplification of the secondary mass are still large, but are of the same order of magnitude of the corresponding values realized by the TMD for $f=1$ (this can be seen by comparing the zoom in the plot in Fig. $6 \mathrm{~b}$ with the plot of Fig. 3a).

The above analyses show that, in general, the displacements of the secondary mass in a MF system strongly increase for lower mass ratios and conversely. A similar effect is produced by a variation of the damping in the connections, namely an increase of the damping reduces the displacements of the secondary mass.

\section{Overtuned Systems}

Increasing the tuning ratio $f$ at fixed mass ratio $\mu$ corresponds to realize stiffer connections between the two masses. This has the effect of shifting the second peak in the resonance curve towards higher and higher frequencies.

In this case, for both TMD and MF systems, the vibration damping efficiency in the proximity of $\rho=1$ decreases, being replaced by an effect of filtering high-frequency excitations.

Figure 7 shows the variation of the performances of both systems for tuning ratios increasing from $f=1.0$ up to $f=3.0$ at fixed mass ratio. This shows that for TMD, the damping of higher frequencies is lower than 0.20 and ends above values of $\rho=3$ (Fig. 7c). On the other hand, the MF systems show an almost complete damping of higher frequencies without upper limits (Fig. 7d).

Figure 8 focuses attention on the case of $f=1.6$ for various mass ratios. In such conditions, the TMD still retains a vibration damping efficiency in a neighborhood of $\rho=1$
Fig. 7 Amplification function $H_{1}(\mathbf{a}, \mathbf{b})$ and vibration damping efficiency $\eta$ (c), $\mu=0.05, \xi_{1}=0.03, \xi_{2}=0.10$. Blue curves correspond to lower values of $f$ d) for $f \in[1.0,3.0]$ with
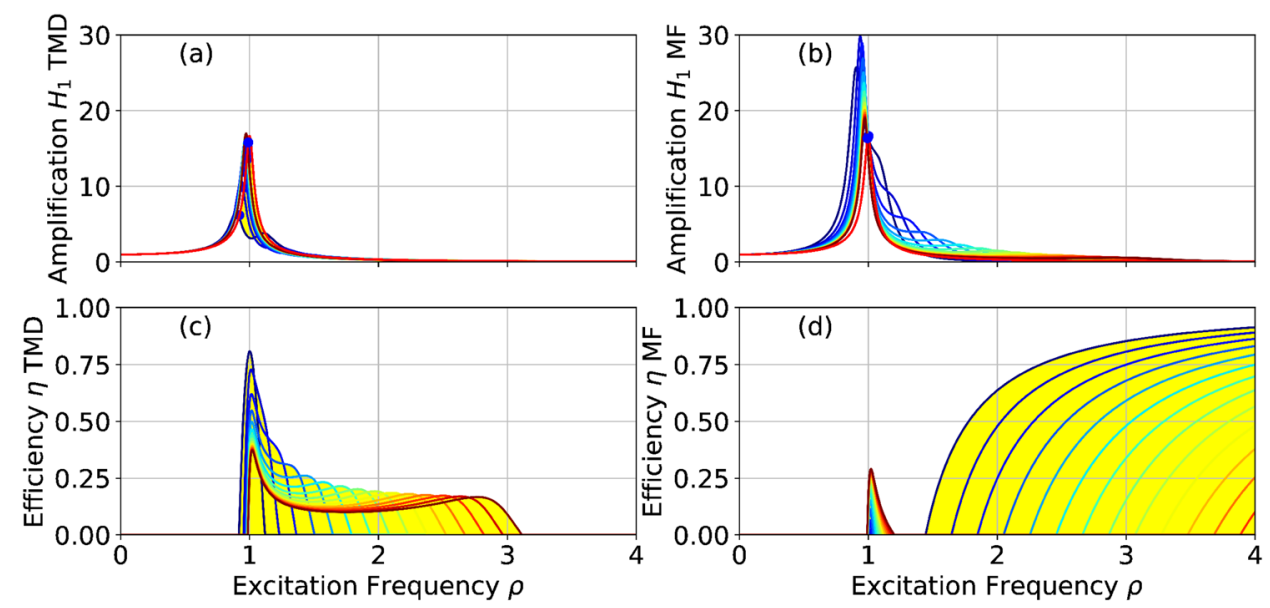

Fig. 8 Amplification function $H_{1}(\mathbf{a}, \mathbf{b})$ and vibration efficiency $\eta(\mathbf{c}, \mathbf{d})$ for $f=1.6$ and various mass ratios $\mu \in[0.05,0.10]$ (other parameters as in Fig. 7) with a zoom of the region $\rho \in[0.8,1.2]$ for the MF system. Blue curves correspond to lower values of mass ratio
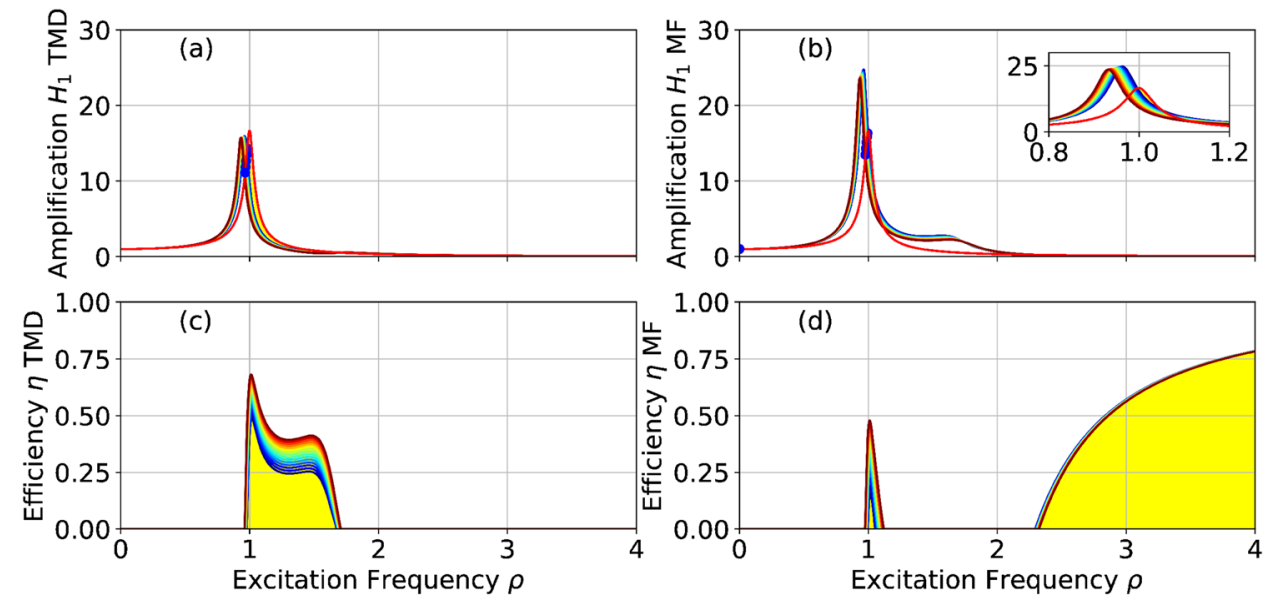
Fig. 9 Amplification factors $H_{2}$ for $f=1.6$ corresponding to Fig. 8. Blue curves correspond to lower values of mass ratio
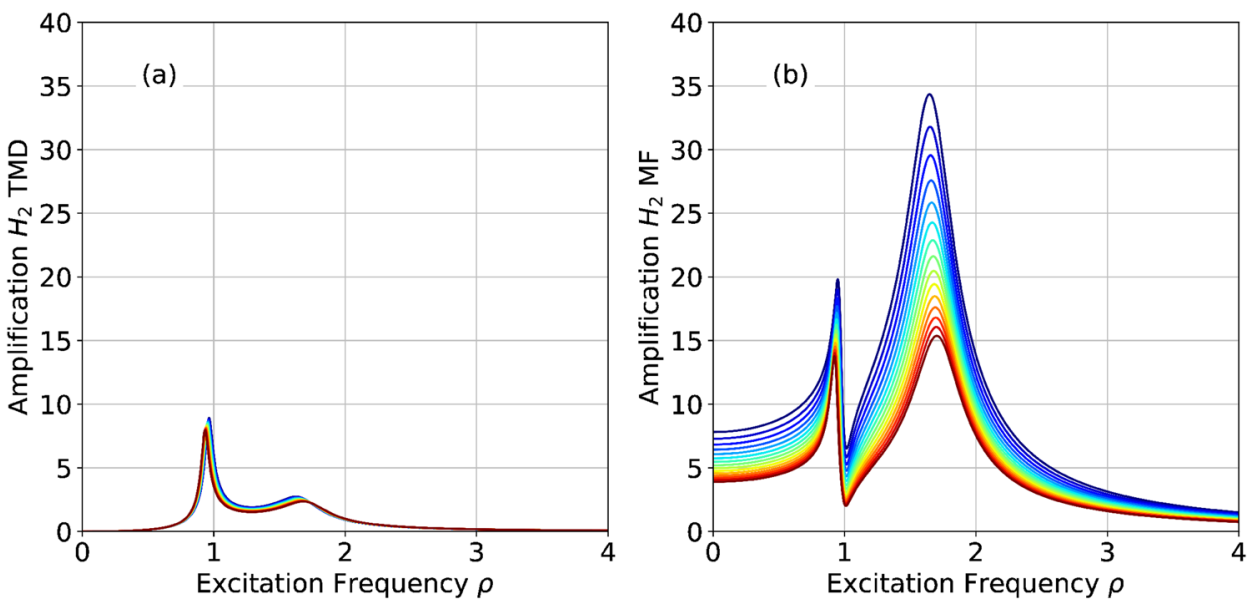

(Fig. 8c) which, however, is lost for higher frequencies. On the contrary, this MF system with stiffer connections has very little efficiency close to $\rho=1$ but has a significant effect of filtering high-frequency excitations (Fig. 8d) and this trend is essentially independent on the mass ratio.

Increasing the stiffness of the connections tends to reduce the oscillations of the secondary mass for both TMD and MF systems as a stronger elastic coupling forces the mass to stay closer and closer. This effect is described by Fig. 9 which shows the transfer functions $\mathrm{H}_{2}$ for $f=1.6$ and highlights how the reduction of the oscillations of the secondary mass induced by the increase of $f$ is further amplified in presence of higher mass ratios (red curves).

\section{Effect of Damping of the Connections}

In previous Sections, the effects of tuning and mass ratios on the response of both systems have been discussed, while keeping constant the value of the damping of both the connection and the main structure. While the damping of the main structure is a peculiarity of the building under consideration, the damping of the connection should be considered as a design variable. The effect of damping in TMD systems is well known. On the other hand, concerning MF systems, it is desirable to endow the connection with enhanced dissipative features. Various types of devices can be conceived for this purpose and the use of high-damping or smart materials, like, for example, friction-based or Shape Memory Alloys might contribute to improve the performances of the MF systems [12, 15, 17].

Focusing attention on MF systems, the increase of damping always entails a significant reduction of the displacements of the secondary structure, typically accompanied by a slight reduction of the vibration efficiency. Figures 10 and 11 illustrate the effect of damping of the connections of a MF system in the two cases of softer and stiffer connections at fixed mass ratio.

Figure 10 shows the case of $f=0.4$ which was considered as representative example of undertuned systems. In this case, an increase of connection damping reduces the displacements of the secondary mass in the region close to
Fig. 10 Effect of connection damping ratio $\xi_{2} \in[0.05,0.60]$ on the performances of undertuned MF systems with $f=0.4$ (other parameters as in Fig. 5. On the left, the vibration damping efficiency, on the right, transfer function $\mathrm{H}_{2}$. Blue curves correspond to lower damping
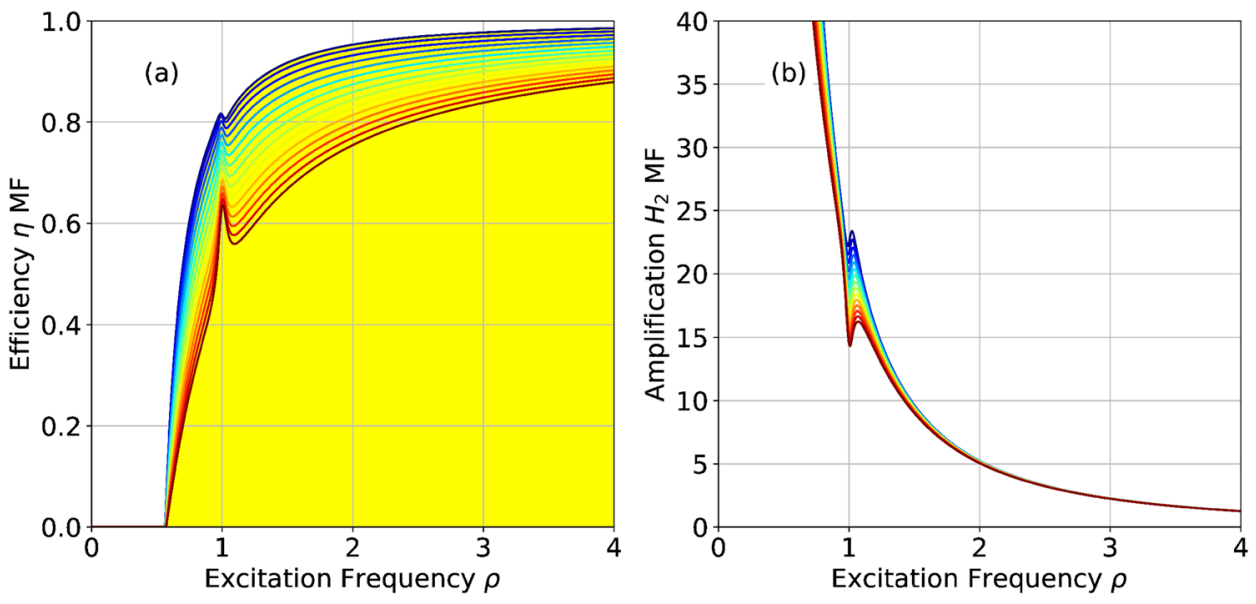
Fig. 11 Effect of connection damping ratio $\xi_{2} \in[0.05,0.60]$ on the performances of overtuned MF systems with $f=1.6$ (other parameters as in Fig. 8). On the left, the vibration damping efficiency, on the right, transfer function $\mathrm{H}_{2}$. Blue curves correspond to lower damping

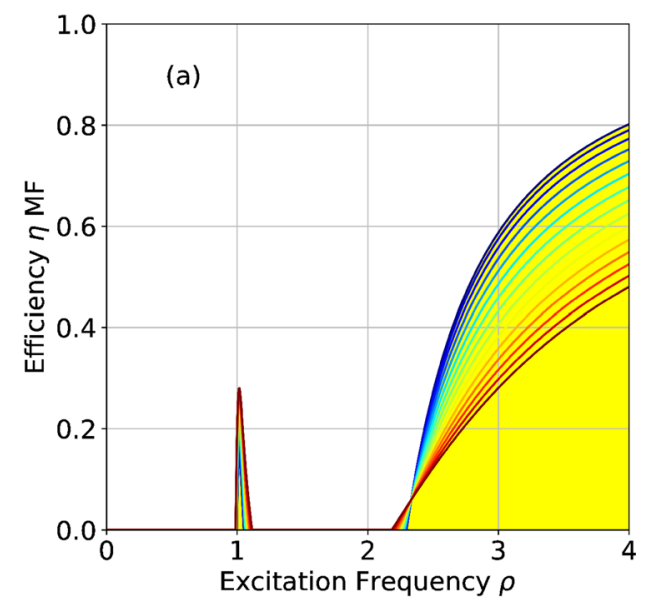

$\rho=1$; while, it has almost no effect in the other excitation frequency ranges.

Figure 11 shows the case of $f=1.6$ which was considered as representative example of overtuned systems. In this case, the increase of damping has a much stronger effect in reducing the displacements of the secondary mass. In both cases, the reduction of the displacements of the secondary is accompanied by a reduction of the vibration damping efficiency.

\section{Conclusions}

With the progress of the façade technology, it is now possible to think of the possibility to realize buildings with cladding systems capable to undergo non-negligible displacements relatively to the main structure. The idea to exploit the vibrations of the façades to improve the performances of the building is appealing, it has been proposed in [8] and it is currently being studied by several authors.

In this work, the performances of buildings with monolithic Moving Façades under harmonic excitations acting on the cladding system have been studied and compared with those of the well-known Tuned Mass Dampers. The comparison has been carried out in terms of the parameter $\eta$ defined in (13) which turns out to be a useful numerical indicator of the vibration damping efficiency.

The results of the comparison show that, although in the case of tuned systems $(f=1)$ TMD definitely shows better vibration damping performances, MF could be potentially very efficient for undertuned systems $(f<0.5)$. However, the good performances of this type of MF could be realized only at the price of extremely large displacements of the façade. The possibility to pursue potential applications of this type of systems seems therefore to be subordinated to the search of solutions to limit such displacements within functionally acceptable ranges.
The results obtained in the comparison also showed that overtuned MF systems may exhibit good performances to isolate the building from high-frequency excitations.

The present comparison has been carried out in the simplest setting of 2 d.o.f. modeling under harmonic excitation focusing attention on the displacement response. Further investigations will be carried out by considering multi-d.o.f. models capable to describe different topological arrangements of the cladding, more realistic excitations and evaluating the vibration efficiency in terms of other structural response parameters like interstory drifts or floor accelerations.

Acknowledgements The authors would like to thank the organizing committee of the 15th International Conference on Vibration Engineering and Technology of Machinery (VETOMAC XV), where part of this work was presented.

The support of Sapienza Grant N. RM11816433E1B0A1 titled "Smart dampers integrated in Double Skin Façades for the mitigation of wind-induced vibrations in tall buildings" is also gratefully acknowledged.

Funding Open access funding provided by Università degli Studi di Roma La Sapienza within the CRUI-CARE Agreement.

\section{Compliance with ethical standards}

Conflict of interest The authors declare that they have no conflict of interest.

Open Access This article is licensed under a Creative Commons Attribution 4.0 International License, which permits use, sharing, adaptation, distribution and reproduction in any medium or format, as long as you give appropriate credit to the original author(s) and the source, provide a link to the Creative Commons licence, and indicate if changes were made. The images or other third party material in this article are included in the article's Creative Commons licence, unless indicated otherwise in a credit line to the material. If material is not included in the article's Creative Commons licence and your intended use is not permitted by statutory regulation or exceeds the permitted use, you will 
need to obtain permission directly from the copyright holder. To view a copy of this licence, visit http://creativecommons.org/licenses/by/4.0/.

\section{References}

1. Shameri MA, Alghoul MA, Sopian K, Zain MFM, Elayeb O (2011) Perspectives of double skin façade systems in buildings and energy saving. Renew Sustain Energy Rev 15(3):1468-1475

2. Boswell CK (2013) Exterior building enclosures: design process and composition for innovative façades. Wiley, New York

3. Wigginton M, Harris J (2013) Intelligent skins. Routledge, Abingdon

4. Aelenei D, Aelenei L, Vieira CP (2016) Adaptive façade: concept, applications, research questions. Energy Procedia 91:269-275

5. Bonham MB (2019) Bioclimatic double-skin façades. Routledge, Abingdon

6. Bedon C, Amadio C (2018) Numerical assessment of vibration control systems for multi-hazard design and mitigation of glass curtain walls. J Build Eng 15:1-13

7. Luible A, Overend M, Aelenei L, Knaack U, Perino M, Wellershoff F (2015) Adaptive facade network-Europe, TU Delft

8. Moon K-S (2009) Tall building motion control using double skin façades. J Arch Eng 15(3):84-90

9. Fu TS, Zhang R (2016) Integrating double-skin façades and mass dampers for structural safety and energy efficiency. J Arch Eng 22:1-12

10. Moon K-S (2017) Integrated design of damping systems for tall buildings. Int J Eng Technol Sci Res 4:1195-1201
11. Pipitone G, Barone G, Palmeri A (2018) Optimal design of double-skin façades as vibration absorbers. Struct Control Health Monit 25:1-16

12. Gong Y, Cao L, Laflamme S, Ricles J, Quiel S, Taylor D (2019) Motion-based design approach for a novel variable friction cladding connection used in wind hazard mitigation. Eng Struct 181:397-412

13. Pinelli J-P, Craig JI, Goodno BJ (1995) Energy-based seismic design of ductile cladding systems. J Struct Eng 121(3):567-578

14. Bedon C, Amadio C (2017) Enhancement of the seismic performance of multi-storey buildings by means of dissipative glazing curtain walls. Eng Struct 152:320-334

15. Gong Y, Cao L, Laflamme S, Ricles J, Quiel S, Taylor D (2019) Variable friction cladding connection for seismic mitigation. Eng Struct 189:243-259

16. Piccirillo V, Bernardini D, Rega G (2017) Optimization of a Pseudoelastic Absorber for Vibration Mitigation. Procedia Eng 199:1779-1784

17. Bernardini D, Rega G (2017) Evaluation of different SMA models performances in the nonlinear dynamics of pseudoelastic oscillators via a comprehensive modeling framework. Int J Mech Sci 130:458-475

Publisher's Note Springer Nature remains neutral with regard to jurisdictional claims in published maps and institutional affiliations. 